Arq. Bras. Med. Vet. Zootec., v.65, n.1, p.203-212, 2013

\title{
Níveis de fósforo disponível em dietas para suínos dos 15 aos $30 \mathrm{~kg}$
}

\author{
[Available phosphorus levels in diets for pigs from 15 to $30 \mathrm{~kg}$ ] \\ J.M. Viana ${ }^{1}$, P.C. Brustolini ${ }^{2}$, F.C.O. Silva ${ }^{3}$, A. Saraiva ${ }^{1}$, J.L. Donzele ${ }^{2}$, R.F.M. Oliveira ${ }^{2}$, A.S. \\ Ferreira $^{2}$, F.A. Santos ${ }^{4}$, E. Paula ${ }^{5}$ \\ ${ }^{1}$ Aluno de pós-graduação - Universidade Federal de Viçosa - Viçosa, MG \\ ${ }^{2}$ Universidade Federal de Viçosa - Viçosa, MG \\ ${ }^{3}$ EPAMIG - Viçosa, MG \\ ${ }^{4}$ Agroceres Nutrição Animal - Rio Claro, SP \\ ${ }^{5}$ Aluno de graduação - Universidade Federal de Viçosa - Viçosa, MG - Bolsista FAPEMIG-Epamig
}

\section{RESUMO}

Avaliou-se a suplementação de dietas com fósforo disponível (Pd) para suínos selecionados para deposição de carne, utilizando-se 100 leitões com média de peso inicial de 15,03 $\pm 0,33 \mathrm{~kg}$, distribuídos em delineamento de blocos ao acaso, com cinco tratamentos, cinco repetições e quatro animais por unidade experimental. Os tratamentos foram dieta basal sem suplementação de fosfato bicálcico e outras quatro dietas obtidas pela suplementação da dieta basal com fosfato bicálcico, resultando em cinco concentrações de $\mathrm{Pd}, 0,106 ; 0,221 ; 0,310 ; 0,406$; e 0,493\%. As porcentagens de Pd influenciaram o consumo diário de ração, que aumentou de forma linear. $\mathrm{O}$ ganho de peso diário aumentou e a conversão alimentar melhorou de forma quadrática até a suplementação máxima de 0,450 e 0,390\% de Pd, respectivamente. Não houve efeito da suplementação das dietas sobre a resistência óssea. As porcentagens de Pd influenciaram de forma quadrática os teores de cálcio e de fósforo nos ossos, que aumentaram até a adição máxima de 0,388 e 0,369\% de $\mathrm{Pd}$, respectivamente. Não houve efeito da porcentagem de Pd sobre o teor de cinza óssea. Suínos dos 15 aos 30kg exigem maior concentração de Pd para máximo ganho de peso $(0,450 \%$ ou $5,33 \mathrm{~g} / \mathrm{dia})$ e melhor conversão alimentar $(0,390 \%$ ou $4,52 \mathrm{~g} / \mathrm{dia})$ em relação às recomendações atuais.

Palavras-chave: desempenho, exigência, fase inicial, minerais, suíno

\begin{abstract}
The supplementation of diets with available phosphorus (aP) for pigs selected for meat deposition was evaluated, using 100 commercial hybrid piglets, 50 castrated males and 50 females, with average initial weight of $15.03 \pm 0.33 \mathrm{~kg}$ allotted in a randomized block design with five treatments, five replicates, and four pigs, 2 males and 2 females per experimental unit. The treatments were a basal diet and other four diets obtained through basal diet supplementation with dicalcium phosphate to obtain five aP concentrations, $0.106,0.221,0.310,0,406$, and $0.493 \%$. The percentages of aP influenced daily feed intake, which increased linearly. Daily weight gain increased and feed conversion improved quadratically up to 0.450 and $0.390 \%$ maximum responses. There was no effect of diet supplementation on bone strength. The percentages of aP influenced the calcium and phosphorus contents in the bones, which increased quadratically up to 0.388 and $0.369 \%$ maximum responses, respectively. There was no effect of aP percentage in the bone ashe content. Pigs from 15 to $30 \mathrm{~kg}$ require greater amount of aP in the diet for maximal weight gain $(0.450 \%$ or $5.33 \mathrm{~g} / \mathrm{d})$ and greatest feed conversion $(0.390 \%$ or $4.52 \mathrm{~g} / \mathrm{d})$ compared to current requirements.
\end{abstract}

Keywords: initial phase, minerals, performance, pig, requirements

Recebido em 21 de junho de 2011

Aceito em 25 de setembro de 2012

*Autor para correspondência (corresponding author)

E.mail: fcosilva@epamig.br 


\section{INTRODUÇÃO}

A formulação de dietas para suínos requer informações precisas das exigências de nutrientes nas diversas fases da criação, de forma a maximizar a deposição de tecido muscular. A deposição de proteína, que é determinada pelo potencial genético, somente ocorrerá de maneira eficiente quando os nutrientes, entre eles o fósforo, forem fornecidos o mais próximo possível das exigências nutricionais dos animais. O fósforo, depois da energia e da proteína, é o nutriente mais caro nas rações para suínos (Shurson e Pomerenke, 2008). Além de sua importância econômica, desempenha papel fundamental na deposição de proteína corporal em razão do seu envolvimento no metabolismo da energia, na síntese de ácidos nucleicos e na estrutura das membranas celulares.

A exigência de fósforo de suínos é dependente da quantidade necessária para suprir as necessidades de mantença dos animais e também da taxa e do tipo de tecido que está sendo depositado. O conteúdo de fósforo no tecido muscular é maior quando comparado ao do tecido adiposo (Wiseman e Mahan, 2010), dessa forma, a necessidade de fósforo disponível para maximizar o ganho de peso dos suínos pode aumentar em função do aumento do potencial genético para deposição de proteína corporal em relação à deposição de tecido adiposo. Como as empresas de melhoramento disponibilizam constantemente no mercado novos materiais genéticos com maior capacidade para produção de carne, estudos para avaliação das exigências de fósforo disponível dessas novas linhagens são e continuarão a ser necessários, a fim de garantir a máxima expressão do potencial produtivo dos animais.

A simples redução da quantidade de fósforo fornecida nas dietas pode resultar em deficiências, ainda que moderadas, e, consequentemente, afetar negativamente a taxa de crescimento e a integridade óssea dos animais. Dessa forma, a primeira abordagem para melhorar a eficiência na utilização do fósforo é garantir um aporte adequado desse mineral em função do potencial genético dos suínos, evitando-se níveis deficientes na ração, medida fundamental para garantir produção eficiente e bem-estar dos animais (Alexander et al., 2008), o que pode ser feito por meio da formulação de dietas com margens de segurança que contribuem para aumentar a excreção desse mineral. Nesse sentido, este estudo foi realizado com o objetivo de avaliar porcentagens de fósforo disponível na dieta sobre o desempenho e as características ósseas de leitões, machos castrados e fêmeas, dos 15 aos $30 \mathrm{~kg}$.

\section{MATERIAL E MÉTODOS}

O experimento foi realizado na creche da granja de suínos da Fazenda Experimental Vale do Piranga, de propriedade da Empresa de Pesquisa Agropecuária de Minas Gerais (EPAMIG), localizada no município de Oratórios - MG, sendo que o projeto foi protocolado como processo 30/2012, no Comitê de Ética para Uso de Animais do Departamento de Zootecnia da UFV.

Foram utilizados 100 leitões híbridos comerciais, 50 machos castrados e 50 fêmeas, com média de peso inicial de $15,03 \pm 0,33 \mathrm{~kg}$, distribuídos em delineamento experimental de blocos ao acaso, com cinco tratamentos, porcentagens de fósforo disponível, cinco repetições e quatro animais, dois de cada sexo, por unidade experimental. A unidade experimental foi representada pela gaiola, e, na formação dos blocos, foi levado em consideração o peso inicial dos leitões.

Os animais foram alojados em gaiolas metálicas $(1,60 \times 1,0 \mathrm{~m})$ com piso e laterais teladas, suspensas $0,56 \mathrm{~m}$ do chão, dotadas de comedouros semiautomáticos e bebedouro tipo chupeta, localizadas em prédio de alvenaria coberto com telhas de cerâmica. As condições ambientais no interior das salas foram monitoradas diariamente, uma vez ao dia (8h), por meio de termômetros de máxima e mínima.

As dietas experimentais (Tab. 1) foram formuladas à base de milho e farelo de soja e suplementadas com minerais e vitaminas, de modo a atenderem às exigências dos animais na fase inicial, 15 a $30 \mathrm{~kg}$, conforme recomendações de Rostagno et al. (2005), exceto o fósforo disponível. Os tratamentos, que consistiram de diferentes porcentagens de fósforo disponível (Pd), foram constituídos de uma dieta basal e outras cinco dietas obtidas pela suplementação da dieta basal com o fosfato bicálcico, em substituição ao calcário calcítico e ao caulim, usado como inerte, resultando em dietas 
experimentais com 0,$106 ; 0,221 ; 0,310 ; 0,406$; e $0,493 \%$ de Pd.

Os aminoácidos industriais foram adicionados, quando necessário, para atender às relações com a lisina digestível preconizadas por Rostagno et al. (2005), segundo o conceito de proteína ideal.
As rações e a água foram fornecidas à vontade aos animais. As rações, as sobras e os desperdícios foram pesados semanalmente, e os animais pesados no início e no final do experimento, que teve duração de 21 dias, para determinação do consumo de ração médio diário (CRD), do ganho de peso médio diário (GPD) e da conversão alimentar (CA).

Tabela 1. Composição centesimal e calculada das dietas experimentais utilizadas para suínos dos 15 aos $30 \mathrm{~kg}$ de peso vivo

\begin{tabular}{|c|c|c|c|c|c|}
\hline \multirow{2}{*}{ Ingrediente } & \multicolumn{5}{|c|}{ Fósforo disponível na dieta (\%) } \\
\hline & 0,106 & 0,221 & 0,310 & 0,406 & 0,493 \\
\hline Milho grão & 63,136 & 63,136 & 63,136 & 63,136 & 63,136 \\
\hline Farelo de soja & 29,247 & 29,247 & 29,247 & 29,247 & 29,247 \\
\hline Óleo soja & 2,371 & 2,371 & 2,371 & 2,371 & 2,371 \\
\hline Calcário & 1,800 & 1,410 & 1,111 & 0,787 & 0,500 \\
\hline Fósforo bicálcico & 0,000 & 0,621 & 1,103 & 1,620 & 2,090 \\
\hline Inerte & 1,225 & 0,994 & 0,811 & 0,618 & 0,435 \\
\hline Sal comum & 0,410 & 0,410 & 0,410 & 0,410 & 0,410 \\
\hline Mistura vitamínica $^{1}$ & 0,300 & 0,300 & 0,300 & 0,300 & 0,300 \\
\hline Mistura mineral $^{2}$ & 0,100 & 0,100 & 0,100 & 0,100 & 0,100 \\
\hline Promotor de crescimento ${ }^{3}$ & 0,150 & 0,150 & 0,150 & 0,150 & 0,150 \\
\hline Nuflor $^{4}$ & 0,200 & 0,200 & 0,200 & 0,200 & 0,200 \\
\hline Probiótico $^{5}$ & 0,400 & 0,400 & 0,400 & 0,400 & 0,400 \\
\hline Promotor de eficiência alimentar ${ }^{6}$ & 0,040 & 0,040 & 0,040 & 0,040 & 0,040 \\
\hline L-Lisina HCL & 0,387 & 0,387 & 0,387 & 0,387 & 0,387 \\
\hline DL-Metionina & 0,098 & 0,098 & 0,098 & 0,098 & 0,098 \\
\hline L-Treonina & 0,126 & 0,126 & 0,126 & 0,126 & 0,126 \\
\hline BHT & 0,010 & 0,010 & 0,010 & 0,010 & 0,010 \\
\hline Total & 100,00 & 100,00 & 100,00 & 100,00 & 100,00 \\
\hline \multicolumn{6}{|c|}{ Composição calculada } \\
\hline Energia metabolizável (kcal/kg) & 3230 & 3230 & 3230 & 3230 & 3230 \\
\hline Proteína bruta $(\%)^{7}$ & 19,24 & 19,24 & 19,24 & 19,24 & 19,24 \\
\hline Lisina digestível $(\%)^{7}$ & 1,145 & 1,145 & 1,145 & 1,145 & 1,145 \\
\hline Met + Cis digestível $(\%)^{7}$ & 0,640 & 0,640 & 0,640 & 0,640 & 0,640 \\
\hline Treonina digestível $(\%)^{7}$ & 0,721 & 0,721 & 0,721 & 0,721 & 0,721 \\
\hline Sódio (\%) & 0,200 & 0,200 & 0,200 & 0,200 & 0,200 \\
\hline Cálcio (\%) & 0,800 & 0,800 & 0,800 & 0,800 & 0,800 \\
\hline Cálcio analisado (\%) & 0,710 & 0,710 & 0,710 & 0,710 & 0,710 \\
\hline $\mathrm{P}$ total analisado $(\%)$ & 0,284 & 0,391 & 0,555 & 0,582 & 0,671 \\
\hline P disponível (\%) & 0,106 & 0,221 & 0,310 & 0,406 & 0,493 \\
\hline Relação cálcio:P disponível & 6,70 & 3,21 & 2,29 & 1,75 & 1,44 \\
\hline
\end{tabular}

${ }^{1}$ Conteúdo/kg de produto: niacina: 13.650mg; vitamina A: 2.800UI; riboflavina: 2.100mg; biotina: 16.56mg; tiamina: 700mg; colina: $126 \mathrm{~g}$; ácido fólico: 420mg; vitamina B12: $11.550 \mathrm{mg}$; vitamina $\mathrm{K} 3: 2.800 \mathrm{mg}$; ácido pantotênico: $7.350 \mathrm{mg}$; vitamina E: $10.500 \mathrm{mg}$; selênio: $136.5 \mathrm{mg}$; vitamina D: $31.050 \mathrm{UI}$; antioxidante: $1.500 \mathrm{mg}$; piridoxina: $700 \mathrm{mg}$; e veículo q.s.p. $1.000 \mathrm{~g}$.

${ }^{2}$ Conteúdo/kg de produto: zinco: $77.999 \mathrm{mg}$; cobre: $15.750 \mathrm{mg}$; magnésio: 41.850mg; cálcio: $98.800 \mathrm{mg}$; cobalto:

$185 \mathrm{mg}$; iodo: $1.470 \mathrm{mg}$; ferro: $26.250 \mathrm{mg}$; e veículo q.s.p. $1.000 \mathrm{~g}$

${ }^{3}$ Promocobre.

${ }^{4}$ Princípio ativo: florfenicol.

${ }^{5}$ Bioplus: Bacillus subtilis, Min.: 1,6x109ufc/g e Bacillus licheniformis, Min.: 1,6x109ufc/g.

${ }^{6}$ Pig Plus 250.

${ }^{7}$ Valores calculados com base nos coeficientes de digestibilidade dos aminoácidos dos ingredientes, de acordo com Rostagno et al. (2005). 
No final do período experimental, após jejum de 12 horas, um animal de cada unidade experimental, com o peso mais próximo da média de peso da baia, foi abatido por sangramento para colheita da pata anterior direita. As patas colhidas foram colocadas em recipiente de alumínio contendo água e foram fervidas, por 25 minutos, visando ao amolecimento da pele e da carne que envolve os ossos para retirada do terceiro osso metacarpiano. Posteriormente, os metacarpos foram levados à estufa ventilada a $65^{\circ} \mathrm{C}$ por um período de 72 horas. Após esse período, os ossos foram submetidos à quebra por flexão, indicadora de resistência óssea, utilizando-se o aparelho Instron Corporation IX Automated Materials Testing System - modelo 4204, no Laboratório de Papel e Celulose do Departamento de Engenharia Florestal da Universidade Federal de Viçosa, segundo metodologia descrita por Teixeira et al. (2005).

Para realização das análises de cálcio, fósforo e cinza nos ossos, os metacarpos foram desengordurados em extrator de Soxhlet por três horas e novamente levados à estufa ventilada a $65^{\circ} \mathrm{C}$, por um período de 24 horas, para, em seguida, serem triturados em moinho tipo bola. A determinação dos teores de cálcio e fósforo das rações, bem como das concentrações de cálcio $(\mathrm{CaO})$, fósforo $(\mathrm{PO})$ e cinza $(\mathrm{CO})$ nos ossos, foi realizada no Laboratório de Nutrição Animal do Departamento de Zootecnia da Universidade Federal de Viçosa, segundo as técnicas descritas por Silva e Queiroz (2004).

As variáveis de desempenho - CRD, GPD e CA - e das características ósseas - CAO, PO e CO foram analisadas utilizando-se os procedimentos para análises de variância e de regressão, contidos no SAEG (Sistema..., 2000).

\section{RESULTADOS E DISCUSSÃO}

Durante o período experimental, as médias das temperaturas mínima e máxima no interior do galpão mantiveram-se em 20,7 $\pm 2,23$ e $24,2 \pm 1,96^{\circ} \mathrm{C}$, respectivamente. Considerando que a faixa ideal de temperatura para suínos na fase inicial de crescimento sugerida por Coffey et al. (2000) está entre 18 e $28^{\circ} \mathrm{C}$, é possível inferir que os animais não foram submetidos a estresse térmico.

O CRD e o consumo de fósforo disponível (CPd) $-\operatorname{Pd}(\%)$ x $\mathrm{CRD}(\mathrm{g}) / 100$ - aumentaram $(\mathrm{P}<0,01)$ de forma linear (Tab. 2), segundo as equações: $\hat{Y}$ $=996,21+419,239 \mathrm{Pd}\left(\mathrm{r}^{2}=0,75\right)$ e $\hat{\mathrm{Y}}=-0,3043+$ $12,4960 X\left(r^{2}=0,99\right)$, respectivamente. De forma semelhante, Saraiva et al. (2009a), ao avaliarem porcentagens de inclusão de Pd entre 0,114 e $0,649 \%$, também constataram efeito linear crescente sobre o CRD de leitões. Variação significativa no CRD de suínos mantidos em ambiente termoneutro dos 15 aos $30 \mathrm{~kg}$, em razão do aumento da porcentagem dietética de $\mathrm{Pd}$, também foi verificada por Alebrante et al. (2011). Traylor et al. (2005) e Saraiva et al. (2009b) não observaram efeito dos níveis de Pd da dieta sobre o CRD de suínos, dos 17 aos 43 e dos 30 aos $60 \mathrm{~kg}$, respectivamente.

No presente estudo, os animais que receberam a ração com os dois menores níveis $(0,106 \mathrm{e}$ $0,221 \%$ de Pd) apresentaram na média e em valor absoluto uma ingestão voluntária de alimento $10,8 \%$ menor em relação à média de CRD dos animais dos demais tratamentos $(0,310 ; 0,406 \mathrm{e}$ $0,493 \%$ de Pd). De forma semelhante, Saraiva et al. (2009a) e Alebrante et al. (2011) constataram redução no CRD de suínos que receberam ração com menor concentração de Pd. Desse modo, pode-se inferir que níveis de fósforo disponível inferiores a $0,221 \%$ podem comprometer o consumo voluntário de ração de suínos em crescimento.

O GPD dos animais aumentou $(\mathrm{P}<0,01)$ de forma quadrática com a adição de $\mathrm{Pd}$ à dieta até a porcentagem máxima estimada de 0,450\% (Fig. 1). Stahly et al. (2000), Arouca (2008), Saraiva et al. (2009a) e Alebrante et al. (2011) também constataram melhora quadrática no GPD de leitões, dos 15 aos $30 \mathrm{~kg}$, em razão do aumento do teor de $\mathrm{Pd}$ da dieta até as porcentagens máximas de 0,$480 ; 0,534 ; 0,443$ e $0,509 \%$ de $\mathrm{Pd}$, respectivamente. 
Níveis de fósforo disponível...

Tabela 2. Valores de desempenho e parâmetros ósseos de suínos que receberam diferentes níveis de fósforo disponível na ração dos 15 aos $30 \mathrm{~kg}$ de peso vivo

\begin{tabular}{lcccccc}
\hline \multicolumn{1}{c}{ Variáveis } & \multicolumn{5}{c}{ Fósforo disponível na dieta (\%) } & CV \\
\cline { 2 - 6 } & 0,106 & 0,221 & 0,310 & 0,406 & 0,493 & $(\%)$ \\
\hline Peso médio final (kg) & 23,54 & 26,58 & 29,04 & 30,43 & 29,67 & 2,88 \\
Consumo de ração (g/dia) $^{1}$ & 1063 & 1035 & 1143 & 1200 & 1184 & 4,40 \\
Consumo de Pd (g/dia) $^{1}$ & 1,13 & 2,29 & 3,54 & 4,87 & 5,84 & 4,94 \\
Ganho de peso (g/dia) $^{2}$ & 406 & 551 & 668 & 731 & 699 & 5,78 \\
Conversão alimentar $^{2}$ & 2,64 & 1,88 & 1,71 & 1,64 & 1,70 & 7,67 \\
Resistência óssea (N) $_{\text {Cálcio no osso (g/kg) }}^{2}$ & 398 & 531 & 441 & 701 & 536 & 17,73 \\
Fósforo no osso (g/kg) $^{2}$ & 135,7 & 173,9 & 146,6 & 194,3 & 162,7 & 6,60 \\
Teor de cinza óssea (\%) $_{\text {Ca:P no osso }}^{3}$ & 62,7 & 87,2 & 69,2 & 97,4 & 77,2 & 7,02 \\
\hline
\end{tabular}

${ }^{1}$ Efeito linear $(\mathrm{P}<0,01)$.

${ }^{2}$ Efeito quadrático $(\mathrm{P}<0,01)$.

${ }^{3}$ Relação calculada de cálcio:fósforo no osso.

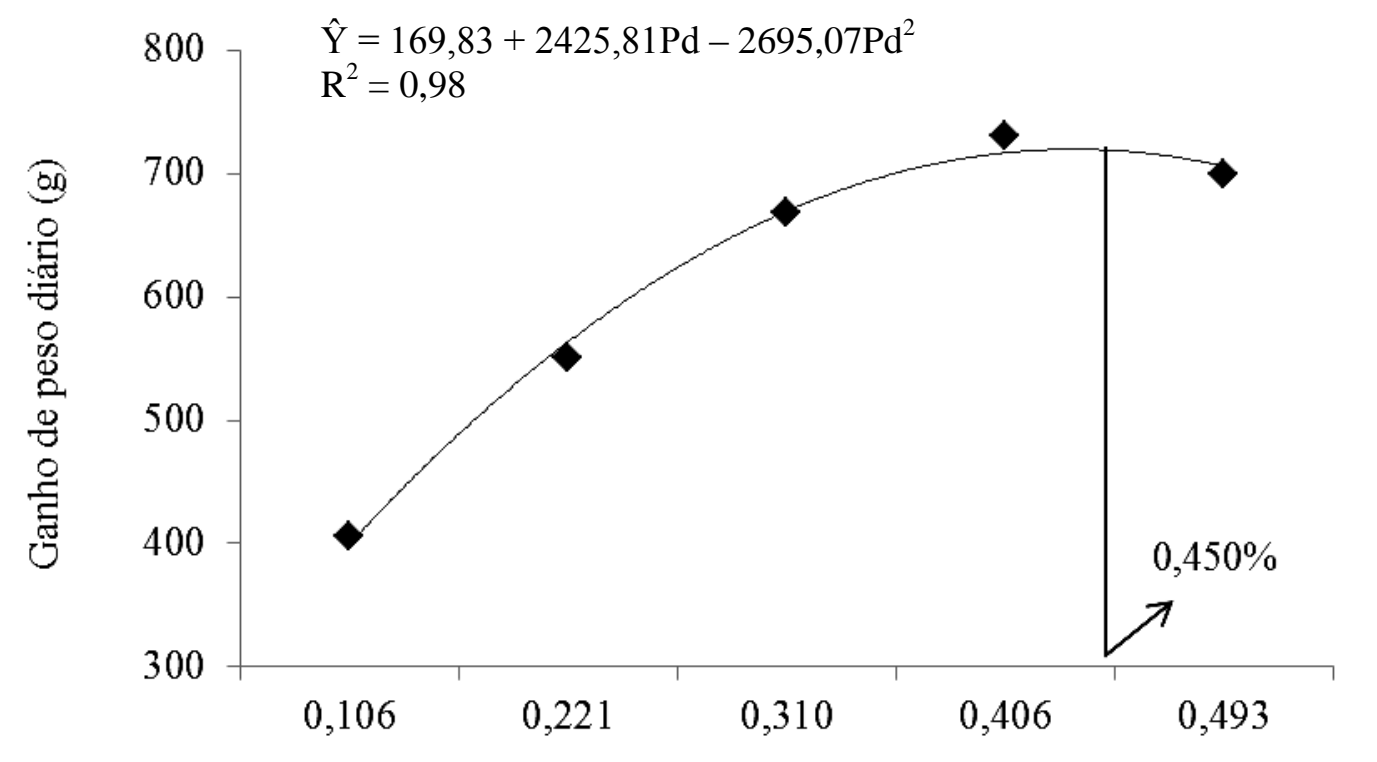

Fósforo disponível na dieta (\%)

Figura 1. Ganho de peso diário de suínos dos 15 aos 30kg, segundo a concentração de fósforo disponível na dieta.

Apesar do padrão de resposta similar para GPD, as diferenças verificadas entre os trabalhos quanto aos níveis de $\mathrm{Pd}$ recomendados que proporcionaram os melhores resultados divergem entre os estudos em razão do potencial genético dos suínos para deposição de tecido muscular (Mahan, 2006; Wiseman et al., 2007). Segundo Wiseman e Mahan (2010), que avaliaram a concentração de minerais no músculo de suínos de duas linhas genéticas, a concentração de fósforo no pernil e no lombo é maior na linhagem de maior potencial para deposição muscular. Os resultados do presente estudo confirmam que as exigências de $\mathrm{Pd}$ dos suínos podem variar em função do potencial genético e que os aumentos verificados na exigência de $\mathrm{Pd}$ de suínos selecionados para maior deposição muscular podem ser em virtude da maior necessidade de fósforo para suportar a síntese proteica. 
A inclusão de $0,450 \%$ de $\mathrm{Pd}$ que proporcionou o melhor resultado de GPD, neste estudo, evidencia que as porcentagens de 0,320 e $0,400 \%$ recomendadas pelo NRC (Nutrient..., 1998) e por Rostagno et al. (2005), respectivamente, podem não ser suficientes para atender às exigências de $\mathrm{Pd}$ das linhagens modernas de suínos disponíveis no mercado, na fase inicial de crescimento, dos 15 aos $30 \mathrm{~kg}$.

Houve efeito $(\mathrm{P}<0,01)$ da porcentagem de $\mathrm{Pd}$ sobre a CA dos leitões (Tab. 2), que melhorou de forma quadrática até a porcentagem máxima estimada de $0,390 \%$ (Fig. 2). De forma semelhante, Arouca (2008), Saraiva et al. (2009a) e Alebrante et al. (2011), ao avaliarem a inclusão crescente de $\mathrm{Pd}$ na dieta de leitões na fase inicial de crescimento, também verificaram melhora de forma quadrática na $\mathrm{CA}$, até a adição estimada de 0,574, 0,477 e 0,461\% de $\mathrm{Pd}$, respectivamente.

Considerando o aumento linear no CRD dos animais no presente estudo, a melhora verificada na CA permite inferir que, além do aumento no GPD até o nível de $0,450 \%$ de Pd, o aumento da concentração de $\mathrm{Pd}$ na dieta pode ter modificado a composição do ganho de peso, favorecendo a maior deposição muscular e a menor deposição de gordura nos animais alimentados com as dietas contendo as maiores concentrações de $\mathrm{Pd}$. Segundo Stahly et al. (2001), dietas deficientes em Pd, além de comprometerem o ganho de peso e a eficiência alimentar dos suínos, podem resultar em maior conteúdo de gordura corporal, influenciando de forma negativa a relação entre a deposição de proteína e de gordura na carcaça.

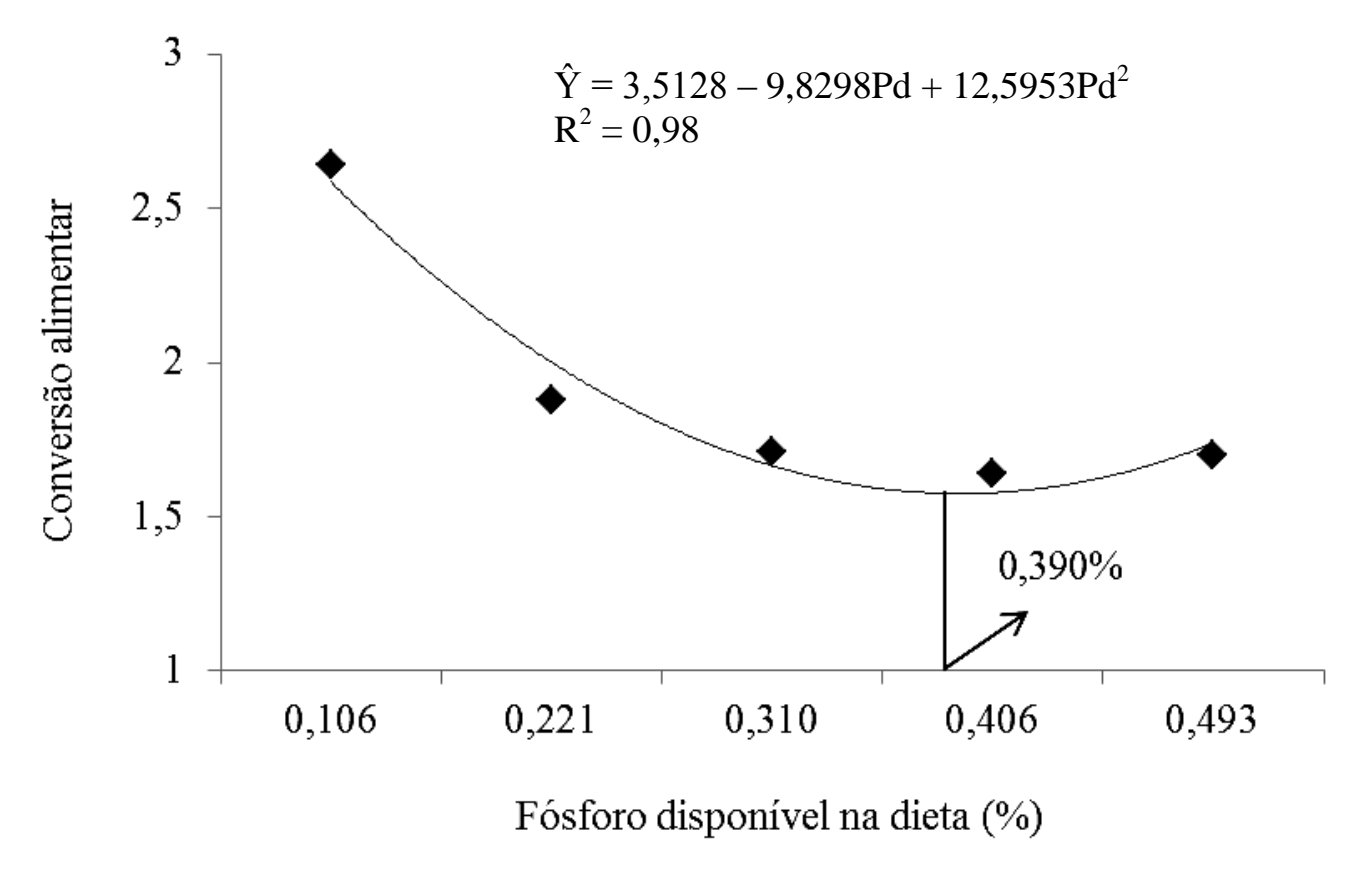

Figura 2. Conversão alimentar de suínos dos 15 aos 30kg, segundo a concentração de fósforo disponível na dieta.

Não foi observado efeito $(\mathrm{P}>0,05)$ da porcentagem de $\mathrm{Pd}$ sobre a resistência óssea (RO) e a cinza óssea (CO) dos animais. Hastad et al. (2004), ao avaliarem porcentagens de $\mathrm{Pd}$ variando entre 0,180 e $0,320 \%$, também não verificaram efeito sobre a $\mathrm{RO}$ e a CO, avaliadas no terceiro e quarto metatarsos. De forma diferente, Arouca (2008) e Saraiva et al. (2009a) constataram efeito linear crescente, enquanto O’Quinn et al. (1997) e Saraiva et al. (2009b) verificaram efeito quadrático sobre a resistência óssea.

As diferenças nos padrões de resposta de RO constatadas entre os trabalhos podem ser atribuídas ao tipo de instrumento utilizado na 
determinação das propriedades físicas dos ossos, aos procedimentos utilizados no preparo das amostras e ao grau de umidade delas, à velocidade de aplicação da força sobre o osso, ao posicionamento do osso no equipamento e, principalmente, ao tipo de osso utilizado (Crenshaw et al., 1981; Saraiva et al., 2009a).

O genótipo dos suínos constitui outro fator que pode estar relacionado com a variação de resultados de resistência óssea de suínos. Hittmeier et al. (2006) observaram que dieta com baixo Pd influencia de forma negativa o GPD somente de animais de genética pura em comparação com suínos de linhagem híbrida. Os autores relataram que os animais puros pareceram priorizar a deposição de fósforo nos ossos em detrimento da taxa de crescimento, o que sugere uma diferença na intensidade do controle homeorrético da utilização do fósforo entre os dois genótipos. Segundo Alexander et al. (2008), diferenças na concentração plasmática de importantes hormônios envolvidos na regulação da homeostase do fósforo ocorrem não somente em função dos níveis de Pd das rações, mas também em função dos diferentes genótipos.

O resultado de $\mathrm{RO}$ obtido no presente estudo não confirmou os obtidos por Saraiva et al. (2009a,b), em que a porcentagem de Pd exigida para máximo ganho de peso foi menor do que a necessária para maximização da resistência óssea. Embora os estudos demonstrem que a exigência de fósforo dos suínos para melhor desempenho (ganho de peso e conversão alimentar) seja maior quando comparada com a porcentagem exigida para máxima mineralização dos ossos, as dietas de suínos, particularmente daqueles destinados à produção de carne, são formuladas para atender às exigências nutricionais para máximo ganho e melhor conversão alimentar (Nutrient..., 1998; Rostagno et al., 2005).

A $\mathrm{CaO}$ e a $\mathrm{PO}$ foram influenciadas $(\mathrm{P}<0,01)$ de forma quadrática pela porcentagem de $\mathrm{Pd}$ da dieta, que aumentou até 0,388 e 0,369\% (Fig. 3 e 4), respectivamente. Saraiva et al. (2009a) e Alebrante et al. (2011) também verificaram efeito quadrático sobre as quantidades de $\mathrm{CaO}$ e PO do terceiro metacarpo com o aumento da concentração dietética de fósforo. Por outro lado, Arouca (2008) constatou que as quantidades de cálcio e fósforo nos metacarpos aumentaram de forma linear crescente com aumento dos níveis de fósforo na ração.

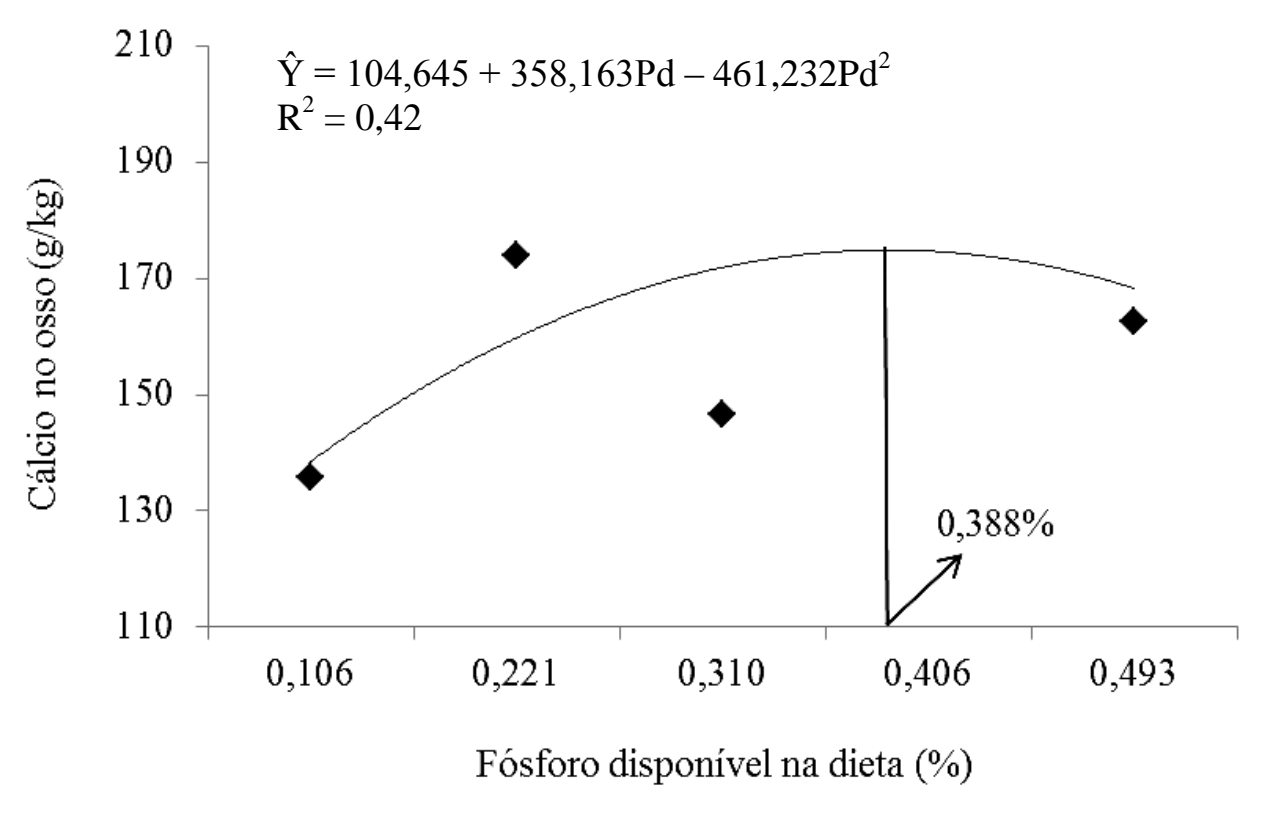

Figura 3. Cálcio nos ossos de suínos dos 15 aos 30kg, segundo a concentração de fósforo disponível na dieta. 


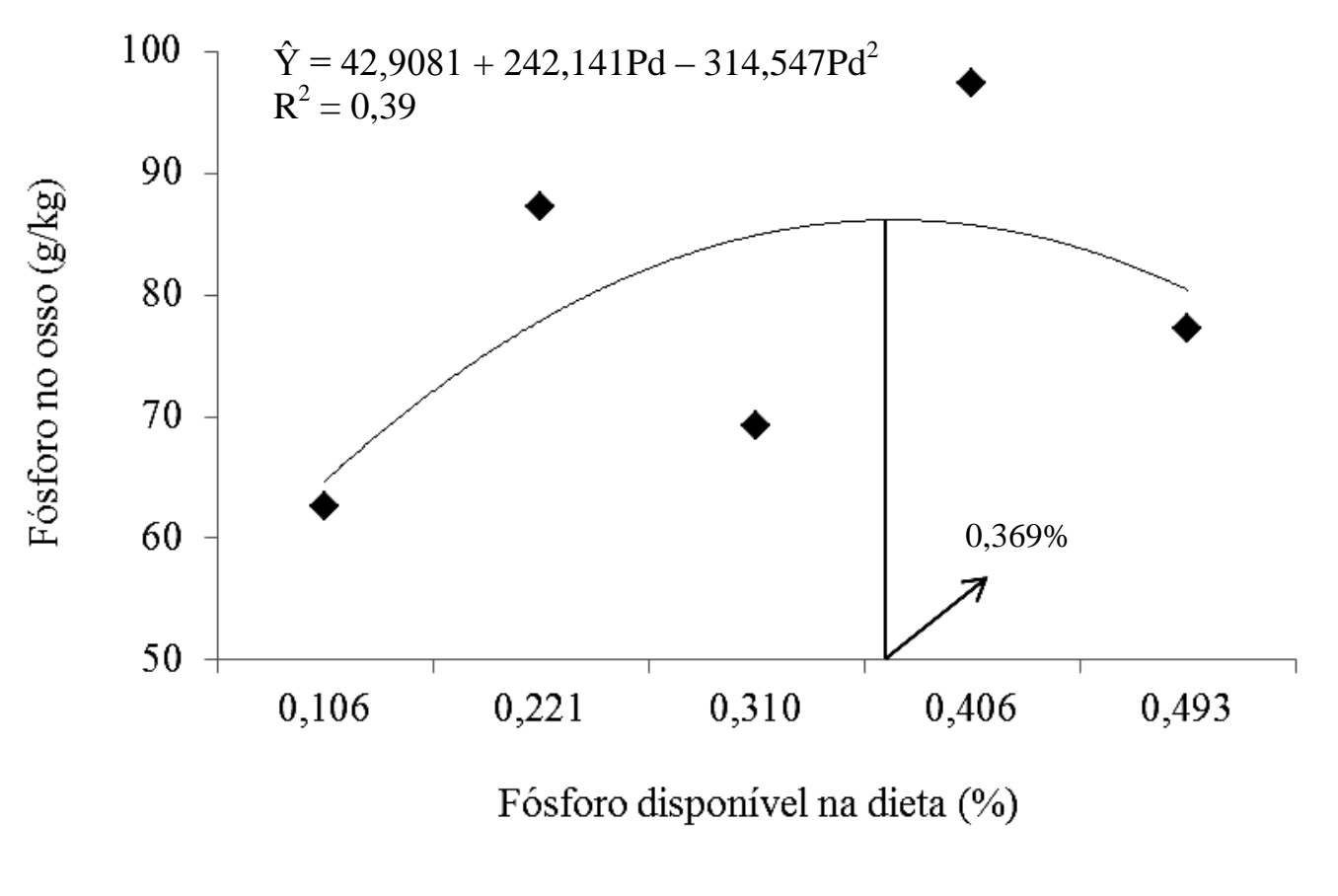

Figura 4. Fósforo nos ossos de suínos dos 15 aos 30kg, segundo a concentração de fósforo disponível na dieta.

Os resultados de $\mathrm{CaO}$ e $\mathrm{PO}$ obtidos no presente estudo confirmam os relatos de Reinhard e Mahan (1986) de que porcentagens de fósforo em excesso na dieta podem ter efeitos negativos sobre as características ósseas de suínos, entretanto não diferem dos resultados de Mahan et al. (1980) e Combs et al. (1990), em que a porcentagem de fósforo exigida para maximizar a mineralização óssea é maior do que a exigida para máximo ganho de peso.

Como a porcentagem de cálcio na dieta, $0,710 \%$, não variou no presente estudo, o aumento verificado na quantidade desse mineral nos ossos, com a elevação das porcentagens de $\mathrm{Pd}$ da dieta, pode ser um indicativo de que as deposições de cálcio e fósforo no osso são interdependentes e tendem a manter uma relação de estreita proporcionalidade de deposição entre eles. Embora a relação cálcio:fósforo disponível (Tab. 1) tenha variado de 6,70 a 1,44 , a relação determinada das quantidades de cálcio e fósforo nos ossos (Tab. 2) variou entre 1,99 e 2,16. Assim, a deposição de cálcio e fósforo nos ossos de suínos parece ocorrer seguindo uma proporcionalidade que independe do conteúdo desses minerais na ração. Fernandéz (1995) também verificou que a relação entre esses dois minerais nos ossos dos animais foi independente do nível de consumo de fósforo e tendeu a ser constante e próxima de 2:1.

A divergência de resultados observada entre trabalhos com relação aos efeitos da adição crescente de Pd sobre as características ósseas de suínos em crescimento pode estar relacionada, sobretudo, ao tipo de osso utilizado nas análises (Crenshaw et al., 1981; Eckhout et al., 1995). A mobilização de fósforo dos ossos ocorre de forma diferente em virtude do tipo de osso. Os ossos esponjosos - costelas, vértebras e externo são os primeiros a serem afetados durante a deficiência de fósforo dietético. Ossos longos, como o úmero, o fêmur, a tíbia, e os ossos pequenos das extremidades do corpo metacarpos e metatarsos, por exemplo - são as últimas reservas a serem usadas. Ketaren et al. (1993) verificaram efeito linear crescente da porcentagem de Pd da dieta sobre a porcentagem de $\mathrm{CO}$ no rádio e no fêmur, sem alteração significativa nos valores dessa variável no quarto metatarso. Da mesma forma, Weeden et al. (1993) verificaram variação significativa nas porcentagens de $\mathrm{CO}$ do fêmur e da primeira 
costela, entretanto os valores de $\mathrm{CO}$ do terceiro e quarto metatarsos não se alteraram com os níveis de fósforo das rações.

\section{CONCLUSÕES}

Suínos dos 15 aos 30kg exigem maior concentração de Pd na dieta para máximo ganho de peso $(0,450 \%$ ou $5,33 \mathrm{~g} / \mathrm{dia})$ e melhor conversão alimentar $(0,390 \%$ ou $4,52 \mathrm{~g} / \mathrm{dia}) \mathrm{em}$ relação às recomendações atuais.

\section{AGRADECIMENTOS}

À Fundação de Amparo à Pesquisa do Estado de Minas Gerais (FAPEMIG), pelo apoio financeiro ao projeto.

\section{REFERÊNCIAS}

ALEBRANTE, L.; DONZELE, J.L.; OLIVEIRA, R.F.M. et al. Available phosphorus levels in diets for pigs with high genetic potential for lean meat deposition kept in thermoneutral environment from 15 to $30 \mathrm{~kg}$. Rev. Bras. Zootec., v.40, p.323$330,2011$.

ALEXANDER, L.S.; QU, A.; CUTLER, S.A. et al. Response to dietary phosphorus deficiency is affected by genetic background in growing pigs. J. Anim. Sci., v.86, p.2585-2595, 2008.

AROUCA, C.L.C. Exigência de fósforo disponível para suínos selecionados geneticamente para deposição de carne em diferentes fases de crescimento, dos 15 aos $120 \mathrm{~kg}$. 2008. 81f. Tese (Doutorado em Zootecnia) - Escola de Veterinária, Universidade Federal de Minas Gerais. Belo Horizonte.

COFFEY, R.D.; PARKER, G.R.; LAURENT, K.M. Feeding growing-finishing pigs to maximize lean grow rate. University of Kentucky. College of Agriculture, 2000. Disponível em: <http://www.animalgenome.org/edu/PIH/prod_g row_finish.pdf $>$. Acessado em: 14 mai. 2010.
COMBS, N.R.; KORNEGAY, E.T.; LINDERMANN, M.D. et al. Calcium and phosphorus requirement of swine from weaning to market weight: II. Development of response curves for bone criteria and comparison of bending a shear bone testing. J. Anim. Sci., v.69, p.682-693, 1990.

CRENSHAW, T.D.; PEO Jr., E.R.; LEWIS, A.S. et al. Bone strength as a trait for assessing mineralization in swine: a critical review of techniques involved. J. Anim. Sci., v.53, p.827835, 1981.

ECKHOUT, W.; PAEPE, M.; WARNANTS, N. et al. An estimation of the minimal $\mathrm{P}$ requirements for growing-finishing pigs, as influenced by the Ca level of the diet. Anim. Feed Sci. Tech., v.52, p.29-40, 1995.

FERNÁNDEZ, J.A. Calcium and phosphorus metabolism in growing pigs. II. Simultaneous radio-calcium and radio-phosphorus kinetics. Liv. Prod. Sci., v.41, p.243-254, 1995.

HASTAD, C.W.; DRITZ, S.S.; TOKACH, M.D. et al. Phosphorus requirements of growingfinishing pigs reared in a commercial environment. J. Anim. Sci., v.82, p.2945-2952, 2004.

HITTMEIER, L.J.; GRAPES, L.; LENSING, R.L. et al. Genetic background influences metabolic response to dietary phosphorus restriction. J. Anim. Sci., v.17, p.385-395, 2006.

KETAREN, P.P.; BATTERHAM, E.S.; WHITE, E. Phosphorus studies in pigs. 1. Available phosphorus requirements of grower/finisher pigs. Br. J. Nutr., v.70, p.249-268, 1993.

MAHAN, D. Necessidades de minerales em cerdos selecionados por um alto contenido em magro y cerdas de alta productividad. FEDNA, 2006. Disponível em: <http://www.etsia.upm.es/fedna/capitulos/06CA P_VIII.pdf $>$. Acessado em: 18 mai. 2010.

MAHAN, D.C.; EKSTRON, K.E; FETTER, A.W. Effect of dietary protein, calcium and phosphorus for swine from 7 to 20 kilograms body weight. J. Anim. Sci., v.50, p.309-314, 1980. 
NUTRIENT requirements of swine. 9.ed. Washington: National Academy of Sciences, 1998. 189p.

O'QUINN, P.R.; KNABE, D.A.; GREGG, E.J. Digestible phosphorus needs of terminal-cross growing-finishing pigs. J. Anim. Sci., v.75, p.1308-1318, 1997.

REINHARD, G.A.; MAHAN, D.C. Effect of various calcium:phosphorus ratios at low and high dietary phosphorus for starter, grower and finishing swine. J. Anim. Sci., v.63, p.457-466, 1986.

ROSTAGNO, H.S.; ALBINO, L.F.T.; DONZELE, J.L. et al. Tabelas brasileiras para aves e suínos; composição de alimentos $e$ exigências nutricionais, 2.ed. Viçosa: UFV, 2005. 186p.

SARAIVA, A.; DONZELE, J.L.; ABREU, M.L.T. et al. Available phosphorus levels in diets for swine from 15 to $30 \mathrm{~kg}$ genetically selected for meat deposition. Rev. Bras. Zootec.,v.38, p.307-313, 2009a.

SARAIVA, A.; DONZELE, J.L.; OLIVEIRA, R.F.M. et al. Níveis de fósforo disponível em rações para leitoas de alto potencial genético para deposição de carne dos 30 aos 60kg. Rev. Bras. Zootec., v.38, p.1279-1285, 2009 b.

SHURSON, J. e POMERENKE, J. Use of US DDGS in practical swine diet formulations. University of Minnesota. International Distillers Grains Conference. 2008. Disponível em: <http://www.thepigsite.com/articles/2629/use-ofus-ddgs-in-practical-swine-diet-formulations $>$. Acessado em: 12 abr. 2011.

SILVA, D.J. e QUEIROZ, A.C. Análise de alimentos: métodos químicos e biológicos. 4. ed. Viçosa: Universidade Federal de Viçosa, 2004. 235p.
SISTEMA de análises estatísticas e genéticas SAEG. Viçosa: UFV, 2000. Versão 8.0.

STAHLY, T.S. Nutrient needs for high lean pigs. Manitoba agriculture, food and rural initiatives. 2001. Disponível em: <http://www.gov.mb.ca/agriculture/livestock/ /pork/swine/bab10s13.html>. Acessado em: 21 jun. 2010.

TEIXEIRA, A.O.; LOPES, D.C.; RIBEIRO, M.C.T. et al. Composição química de diferentes fontes de fósforo e deposição de metais pesados em tecidos de suínos. Arq. Bras. Med. Vet. Zootec., v.57, p.502-509, 2005.

TRAYLOR, S.L.; CROMWELL, G.L.; LINDEMANN, M.D. Bioavailability of phosphorus in meat and bone for swine. J. Anim. Sci., v.83, p.1054-1061, 2005.

WEEDEN, T.L.; NELSSEN, J.L.; GOODBAND, R.D. et al. Effects of porcine somatotropin and dietary phosphorus on growth performance and bone properties of gilts. J. Anim. Sci., v.71, p.26742682, 1993.

WISEMAN, T.G.; MAHAN, D.C. Partition of minerals in body components from high- and low-lean genetic line of barrows and gilts from 20 to 25 kilograms of body weight. J. Anim. Sci., v.88, p.3337-3350, 2010.

WISEMAN, T.G.; MAHAN, D.C.; PETERS, J.C. et al. Tissue weights and body composition of two genetics lines of barrows and gilts from twenty to one hundred twenty-five kilograms of body weight. J. Anim. Sci., v.85, p.1825-1835, 2007. 